\title{
(RE)Organização da Atenção Primária à Saúde para o enfrentamento da COVID-19: Experiência de Sobral-CE
}

\author{
Marcos Aguiar Ribeiro, David Gomes Araújo Junior, Ana Suelen Pedroza Cavalcante, Adriano \\ Ferreira Martins, Larisse Araújo de Sousa, Regina Célia Carvalho, Isabel Cristina Kowal Olm \\ Cunha
}

\section{RESUMO}

O objetivo deste artigo é sistematizar as ações de (re)organização da Atenção Primária à Saúde disparadas pela Secretaria Município de Sobral (CE) no enfrentamento da COVID-19. A (re)organização dos serviços de APS em Sobral centraram-se no desenvolvimento de ações de territorialização e vigilância em saúde para bloquear e reduzir o risco de expansão da epidemia; a continuidade das ações próprias na sua rotina de promoção da saúde, prevenção de agravos e provisão de cuidados à população e o compartilhamento do cuidado em rede. Assim, a experiência de Sobral evidencia a importância do fortalecimento da APS, em especial do modelo assistencial ancorado na abordagem familiar e comunitária.

Palavras-chave: COVID-19; Atenção Primária à Saúde; Vigilância em Saúde.

\section{ABSTRACT}

The objective of this article is to systematize the actions of (re)organization of Primary Health Care launched by the Municipality of Sobral (CE) in the fight against COVID-19. The (re)organization of PHC services in Sobral focused on the development of territorialization and health surveillance actions to block and reduce the risk of spreading the epidemic; the continuity of its own actions in its routine of health promotion, disease prevention and provision of care to the population and the sharing of care in the network. Thus, Sobral's experience shows the importance of strengthening PHC, especially the care model anchored in the family and community approach.

Keywords: COVID-19; Primary Health Care; Health Surveillance.
Revista da Rede APS 2020

Publicada em: 09/06/2020

DOI:10.14295/aps.v2i2.96

Marcos Aguiar Ribeiro (Secretaria Municipal de Saúde de Sobral, Sobral, CE, Brasil; Universidade Federal de São Paulo, São Paulo, SP, Brasil)

David Gomes A. Junior Regina Célia Carvalho (Secretaria Municipal de Saúde de Sobral, Sobral, CE, Brasil; Universidade Federal do Ceará, Fortaleza, CE, Brasil)

Larisse Araújo de Sousa (Secretaria Municipal de Saúde de Sobral, Sobral, CE, Brasil)

Isabel Cristina K. O. Cunha (Universidade Federal de São Paulo; São Paulo; São Paulo; Brasil)

Ana Suelen P. Cavalcante Adriano Ferreira Martins (Universidade Federal do Ceará, Fortaleza, CE, Brasil)

Correspondência para:

Marcos Aguiar Ribeiro marcosribeiroce@gmail.com 


\section{INTRODUÇÃO}

A pandemia causada pelo novo coronavírus devido a rápida transmissão sustentada nos continentes (WHO, 2020; GORBALENYA et al., 2020) tem exigido que os serviços de saúde de todo o mundo se (re)organizem para buscar atender as necessidades de saúde da população.

Um dos focos foi a ampliação de leitos hospitalares, principalmente no que diz respeito aos de Unidades de Terapia Intensiva, visto que as infecções pelo novo coronavírus podem evoluir para quadros mais graves como a Síndrome Respiratória Aguda Grave (TUÑAS et al., 2020; ZHANG et al, 2020; BRASIL, 2020a).

Além disso, a Atenção Primária à Saúde também exerce papel central no enfrentamento da COVID-19, uma vez que está ancorada nos princípios de proteção da saúde, na prevenção e controle de doenças, sendo capaz para colaborar estrategicamente com a redução do risco de transmissão da doença a partir do diagnóstico precoce, acompanhamento e monitoramento individual e familiar (BARBOSA; SILVA, 2020).

As características implícitas do novo coronavírus, tais como período de incubação, capacidade de contágio, canais de transmissão de infectados sintomáticos e assintomáticos fazem com que a doença se espalhe rapidamente, em um contexto agravado pela inexistência de tratamento convencional (CASCELLA, 2020), o que mostra ainda mais a importância da APS neste contexto de colaboração para a redução da disseminação do novo coronavírus.

Vale salientar ainda que a propagação da COVID19 que aconteceu rapidamente na China e, se espalhou para outros lugares, principalmente na Europa, nos Estados Unidos, no Canadá e no Brasil (BRASIL, 2020a), fizeram o Ministério da Saúde (MS) decretar o Estado de Emergência em Saúde Pública de Importância Nacional (ESPIN) por meio da Portaria no 188/2020 (BRASIL, 2020b). E a Organização Mundial de Saúde
(OMS) declarar condição de transmissão pandêmica da doença e em março de 2020 e também o estado de transmissão comunitária em todo o Brasil, conforme Portaria no 454 de 20 de março de 2020 (BRASIL, 2020c).

No âmbito do estado do Ceará, o Governo Estadual publicou o Decreto no 33.510, de 16 de março de 2020, adotando significativas medidas de contingenciamento da pandemia (CEARÁ, 2020a). Em Sobral, diante da responsabilidade municipal no enfrentamento ao novo coronavírus, a Prefeitura Municipal decretou em 13 de março de 2020 (Decreto no 2.369/2020) estado de perigo público e em 16 de março de 2020, estado de Emergência municipal, conforme Decreto no 2.371 (SOBRAL, 2020a; SOBRAL, 2020b). A partir de então, Sobral que tem como um de seus pilares da atenção a Estratégia Saúde da Família optou por traçar ações estratégicas neste âmbito para o enfrentamento da COVID-19.

A epidemia do novo coronavírus exige a invenção criativa de novos modos de cuidados que devem estar aliados a reafirmação dos atributos da APS e dos princípios doutrinários e organizativos do Sistema Único de Saúde, sendo a Estratégia Saúde da Família o modo mais efetivo de se fazer APS, requerendo a contratação emergencial de equipes de SF e a revitalização dos Núcleos de Apoio à Saúde da Família (NEDEL, 2020).

Neste sentido, é importante que as experiências nos diferentes cenários sejam compartilhadas para que possam ser refletidas e até mesmo replicadas/repensadas em busca da contenção da disseminação do novo coronavírus e da efetivação da APS, no Brasil. Além disso, sistematizar experiências nesse momento de crise é capaz de trazer mais evidências da importância do SUS como projeto ético político de uma sociedade que valoriza a vida.

Neste contexto, o objetivo deste artigo é sistematizar as ações de (re)organização da Atenção Primária à Saúde disparadas pela 
Secretaria Município de Sobral (CE) no enfrentamento da COVID-19

\section{SOBRAL: DO INÍCIO DOS CASOS À CRIAÇÃO DO GRUPO DE CONDUÇÃO DA}

\section{PANDEMIA}

Em janeiro de 2020, antes do início da transmissão comunitária de COVID-19 no Brasil, Sobral teve um primeiro caso provável importado, posteriormente descartado. O surgimento prematuro desse caso suspeito alertou a secretária municipal de saúde e a partir do mês de fevereiro foi iniciado um processo de reorganização da rede para o enfrentamento de uma possível onda de casos de COVID-19 onde foi disparado a produção do plano de contingência do município.

Diante o primeiro possível caso importado de COVID-19 no município, a vigilância epidemiológica e APS foram acionadas no intuito de elaborar um plano de ação de rastrear a linha do tempo com intuito de identificar a possibilidade de outras contaminações no município. Em conjunto a vigilância e a APS iniciaram o monitoramento dos contatos do possível caso até a divulgação do resultado descartado, orientado quanto ao isolamento domiciliar e identificação precoce de sintomas sugestivos da COVID-19.

Após esse episódio e diante a proporção do aumento de casos de COVID-19 no mundo acompanhado pelos documentos oficiais da OMS a secretaria de saúde com apoio de sua coordenação de políticas e planejamento iniciaram o desenvolvimento de um plano de contingência para o município, onde os serviços de saúde e células da secretária apresentaram ações de enfretamento a COVID-19 de acordo com sua realidade e seu público alvo de atendimento. Algumas series de reuniões em parceria com diretores e gerentes dos estabelecimentos foram realizados no intuito de alinhas as ações em rede e coordenador todo sistema de saúde em prol do enfretamento a contaminação do vírus pelos usuários do sistema.

Como acompanhado pelos documentos oficiais da OMS e ministério da saúde a contaminação da COVID-19 não pode ser contida no mundo e nem muito menos no Brasil, portanto ações precisaram ser revista diante o anúncio de contaminação comunitária divulgado pelo governo do estado do Ceará em março de 2020. No que concerna a atual situação foi criado um Centro de Operações Estratégicas em Saúde Pública, no qual participam representantes das secretárias do município, universidades públicas e privadas, das indústrias, do terceiro setor, do setor saúde, dos hospitais públicos e privados. Esse Centro tem facilitado a implementação de ações intersetoriais e propiciado a reflexão contínua sobre as decisões, contribuindo no planejamento de intervenção e apoiando o município no que se refere a evidências científicas e na tomada de decisões para melhor manejo da crise em saúde público com intuito de minimizar os efeitos da pandemia em todos os setores e cidadãos sobralenses.

O Centro de Operações de Emergências em Saúde Pública de Sobral (COESP-S) é uma das estratégias municipais adotadas em consonância com a Secretaria do Estado e o Ministério da Saúde. Foi legitimado por meio de Portaria municipal. Este Centro é o responsável pela coordenação das ações de resposta às emergências em saúde pública, incluindo a mobilização de instituições, órgãos, profissionais e demais recursos para o restabelecimento dos serviços de saúde e a articulação entre as esferas de gestão do SUS, sendo constituído por profissionais das coordenações-gerais e áreas técnicas da Vigilância em Saúde da Secretaria Municipal da Saúde e da Superintendência Regional em Saúde, bem como de gestores de outras instituições envolvidas na resposta e com competência para atuar na tipologia de emergência identificada.

A Secretária da Saúde do Município é a responsável pela ativação do COESP-S, com base 
no parecer técnico emitido pela Coordenação de Vigilância em Saúde Municipal, definindo quando o evento representar risco à saúde pública seja pela probabilidade de propagação municipal ou pela superação da capacidade de resposta local. Deverá ativar conforme o nível de organização à resposta (Se alerta, iminência de perigo ou emergência de Saúde Pública). Deve responder de forma oportuna e proporcional às situações de emergência em saúde pública, realizando o planejamento, organização, coordenação, avaliação e execução das ações de resposta.

Umas das ações incentivada pelo COESP-S, foi organização de um grupo de profissionais da saúde com a responsabilidade técnica na qual foi nomeado de Comitê de Crise de Enfrentamento a COVID-19 sendo estruturado no âmbito da saúde, com profissionais estratégicos dentro do organograma da secretária municipal da saúde (Secretária Municipal da Saúde; Coordenador Administrativo Financeiro; Coordenadora Jurídica; Coordenadora da Atenção Primária à Saúde; Gerente da Atenção Primária à Saúde; Coordenadora da Atenção Especializada à Saúde; Coordenador de Vigilância do Sistema de Saúde; - Assistente Especial da Atenção Primária à Saúde; Gerente da Célula de Auditoria e Regulação; Gerente de Imunização e Técnicos de Vigilância em Saúde). Tendo como atribuição a gestão e orientação das medidas estratégicas de enfretamento ao novo coronavírus, buscando também planejar ações que visão estabelecer prioridades de acordo as necessidades de saúde locais e realizar reuniões diárias e alinhamentos com os demais trabalhadores da saúde e setores da Prefeitura de Sobral.

\section{A (RE)ORGANIZAÇÃO DA APS}

Sobral é um município localizado no interior do estado de Ceará, a 235 quilômetros da capital Fortaleza. Tem uma população de 208.935 habitantes segundo a estimativa do IBGE para 2019. O município além da zona urbana possui zonas rurais, com distâncias superiores a $60 \mathrm{~km}$ da sede do município.

$\mathrm{Na}$ organização regional do SUS, Sobral configura-se como uma referência para Macrorregião Norte do estado do Ceará composta por 55 municípios, onde se concentra maior parte dos serviços de média e alta complexidade dessa macrorregião. No tocante à APS, são 37 Centros de Saúde da Família (70 equipes de Estratégia Saúde da Família, 6 equipes de NASF, 50 equipes de saúde bucal, 3 equipes multiprofissionais de Atenção domiciliar e 2 academias da saúde), dos quais 23 encontram-se na zona urbana e 14 na zona rural.

No município, a APS é prioritária, sendo realizados importantes esforços para ampliação da cobertura da ESF, melhoria na infraestrutura das unidades e na organização dos processos de trabalho, com o fortalecimento da articulação com os pontos da rede de atenção no intuito de reorientar o modelo de atenção à saúde tendo a APS como protagonista na ordenação e coordenação das redes de atenção no município.

Neste contexto, todo esse processo de fortalecimento da APS tem sido fundamental para o enfrentamento à COVID-19. Assim, a partir do planejamento coletivo do COESP e Comitê de Crise, a APS de Sobral (re)organizou suas práxis com mudanças/adaptações nos serviços e ações ofertados aos usuários do SUS.

Todos os 37 CSF permaneceram abertos e em funcionando, destes 14 CSF ampliaram o horário de atendimento até o turno noturno, com o objetivo de garantir o acesso e qualificar o cuidado de pacientes suspeitos e confirmados da COVID-19.

No entanto, foram desenvolvidas estratégias para evitar as aglomerações e impedir possíveis contágios. Os investimentos realizados nos últimos anos na infraestrutura das unidades têm permitido adaptações da estrutura física para o enfrentamento da COVID-19. Em geral, as unidades apresentam amplo espaço e ventilação, com pelo menos duas entradas 
distintas, espaços para atividades coletivas e estacionamento, o que facilita a construção de fluxos de entrada.

Dadas essas estruturas diferenciadas, foi possível organizar entradas diferentes para os usuários sintomáticos respiratórios e para as outras demandas. Para qualificar o processo de triagem de sintomáticos respiratórios, foram montadas tendas na frente ou nos pátios das unidades para que as equipes realizem a avaliação dos usuários nesses espaços, de modo que os sintomáticos respiratórios evitem ingressar nos consultórios fechados e climatizados que não têm a estrutura adequada para evitar o risco de contágio.

A (re)organização dos serviços de APS no município de Sobral centraram-se em três eixos de intervenção para adequarem-se ao enfrentamento da pandemia, a saber: o desenvolvimento de ações de territorialização e vigilância em saúde para bloquear e reduzir o risco de expansão da epidemia; a continuidade das ações próprias na sua rotina de promoção da saúde, prevenção de agravos e provisão de cuidados à população e o compartilhamento do cuidado em rede.

Assim, com vistas a buscar compreender a APS em uma perspectiva sistêmica no processo de enfrentamento da COVID-19, reflete-se o fazer saúde da Saúde da Família a partir de um mosaico de ressignica(AÇÕES) das práticas cotidianas. Desde modo, utilizou-se a mandala como símbolo para representar de forma esquemática a discussão que será sedimentada neste material, conforme apresentado na Figura 1.

Figura 1 - Representação esquemática do enfrentamento da COVID-19 no contexto da APS de Sobral, Ceará, Brasil (2020).

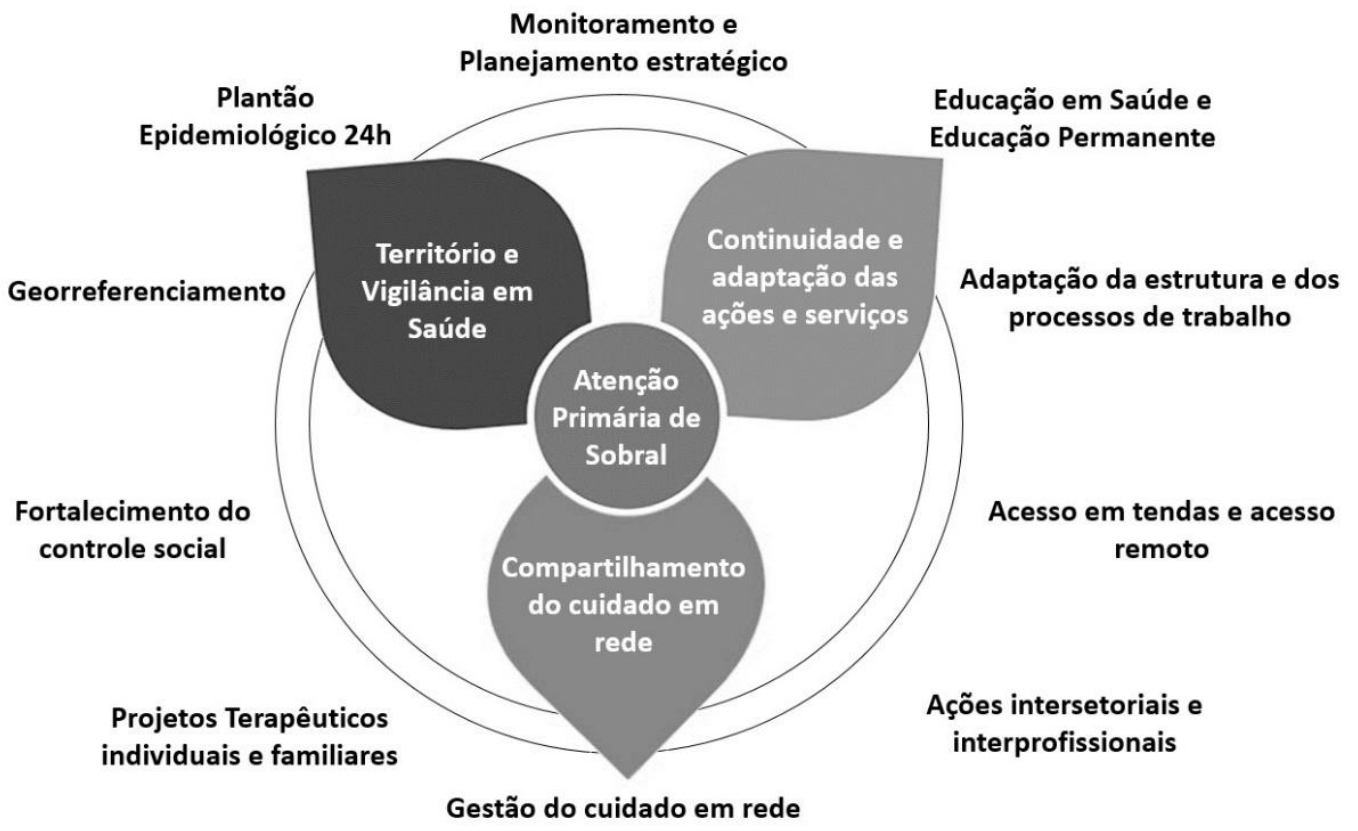

Fonte: Elaborado pelos autores 


\section{AÇÕES DE TERRITORIALIZAÇÃO E VIGILÂNCIA EM SAÚDE}

Fazer saúde da família é saber se (des)territorializar de forma constante em busca de acompanhar a dinamicidade da realidade da população. O território assim como o trabalho é vivo em ato (MERHY, 2002).

Neste sentido, como um dos dispositivos criados a partir da prática de (re)invenção criativa foi implantado o Plantão Epidemiológico 24h, permitindo colaborar na operacionalização de um sistema de vigilância em saúde a nível local (OLIVEIRA; CASANOVA, 2009). As ações da Vigilância em Saúde buscam estar integradas e coordenadas com as realizadas pelos demais dispositivos da Rede Integral de Atenção à Saúde (BRASIL, 2013) do município a fim de proporcionar informações que subsidiarão o planejamento de ações que possam ser realizadas para a implementação de medidas contra o novo coronavírus.

O Plantão Epidemiológico constitui-se como um canal de diálogo entre os usuários do SUS que a partir de aplicativo de mensagens, com vários números, que funciona 24 horas, pode tirar as dúvidas com o profissional que atende a ligação. Este profissional posteriormente ao contato com usuário se comunica com o gerente do CSF onde a pessoa está cadastrada e apresenta o caso. Em seguida, o gerente junto com a equipe estabelece uma estratégia de monitoramento, que pode incorporar a visita domiciliar ou contatos remotos, de acordo com as singularidades de cada caso.

Além disso, este canal de comunicação articulase também com a Central de Monitoramento da COVID-19 e o Comitê de Crise que compartilham situações específicas de usuários com os gerentes da APS, que planejam e desenvolvem juntos às equipes estratégias singulares de monitoramento e cuidado aos usuários inseridos nos territórios.

Os Agentes Comunitários de Saúde (ACS) são atores estratégicos para realizar esse monitoramento nos territórios. Esses profissionais planejam e realizam as visitas domiciliares às famílias de seu território de abrangência. Nestas visitas, realizam orientações acerca do isolamento domiciliar, identificação precoce de sinais de gravidade da doença, sobre as medidas de preventivas para evitar a disseminação do novo coronavírus e também tem o olhar atento (ROLNIK, 2014) a outras demandas de saúde da população. Os Agentes de Combates as Endemias (ACE) também permanecem nos territórios na prevenção das arboviroses.

Estes profissionais se paramentam não só com os Equipamentos de Proteção Individual (EPS), mas também com os conhecimentos (des)construídos e compartilhados em processos formativos relacionados à COVID-19 para realizarem as visitas domiciliares. As visitas antes realizadas dentro dos municípios ampliam seu espaço para as calçadas, estabelecendo um distanciamento de 2 metros entre o profissional e a pessoa a ser cuidada. Estas visitas estão sempre ancoradas em diálogos construtivos de respeito ao saber da população, escuta acolhedora e empatia.

\section{CONTINUIDADE DAS AÇÕES PRÓPRIAS}

\section{NA SUA ROTINA DE PROMOÇÃO DA}

\section{SAÚDE, PREVENÇÃO DE AGRAVOS E PROVISÃO DE CUIDADOS À POPULAÇÃO}

No contexto da pandemia da COVID-19, os profissionais de saúde que integram a APS precisaram adequar seus serviços e ações, de modo a incorporar o atendimento remoto e a descentralização das ações, a fim de fortalecer a abordagem familiar e a comunitária e assim buscar diminuir a disseminação do vírus nos territórios.

Dentro da reorganização da rede de atenção para o enfrentamento à pandemia muitos serviços de da atenção especializada foram suspensos. Os profissionais desses centros de 
especialidades médicas, centros de referência de reabilitação foram realocados para fortalecer a APS. Com esses profissionais foram organizadas novas equipes nas UBS, as quais têm seu território de referência e são responsáveis pelo acompanhamento e monitoramento dessas populações e pela oferta das ações próprias da APS, tais como:

\section{Cuidado a saúde da mulher e da criança}

$\mathrm{O}$ acompanhamento às gestantes, puérperas e crianças é amplificado por meio de uma estratégia conhecida como "Trevo de quatro folhas". Esta estratégia é uma política pública municipal que contempla a participação de "mães sociais", mulheres da comunidade que acompanham todo o processo de gestação e puerpério, desde o território até a maternidade, dando suporte e orientação às famílias em situação de vulnerabilidade. Além disso, propõe cuidado domiciliar para os bebês prematuros com visitas diárias dos ACS e visitas semanais de uma equipe multiprofissional composta por enfermeira pediátrica, médica pediátrica e assistente social. A estratégia tem sido reconhecida em diferentes locais e tem contribuído a reduzir drasticamente a taxa de mortalidade infantil no município.

Com a chegada da pandemia da COVID-19 o prénatal foi suspenso, mas posteriormente reconheceu-se a necessidade de retomar esse cuidado, porém, sem o acompanhamento das "mães sociais" da Estratégia Trevo de Quatro Folhas. No entanto, por meio da estratégia "Trevo de quatro folhas" as gestantes e puérperas já se encontravam mapeadas e monitoradas, o que facilitou a retomada do prénatal e acompanhamento do desenvolvimento da gestação.

Neste momento, o acompanhamento pré-natal é realizado com algumas limitações e maiores cuidados, tais como a redução do número de atendimentos de modo que as consultas de prénatal são agendadas nos territórios com todos os cuidados necessários para evitar contágio, além disso, foram incorporadas teleconsultas para ampliar o monitoramento dos casos.
As ultrassonografias obstétricas foram mantidas, todavia, potencializadas por meio da utilização de ultrassonógrafo portátil e o prénatal de alto risco foi mantido por meio do matriciamento do médico obstetra com o médico de família e comunidade, estes profissionais discutem os casos das gestantes de alto risco e definem o projeto terapêutico.

\section{Imunização}

A imunização e o monitoramento do calendário vacinal encontram-se mantido. No caso dos idosos, a vacinação contra a influenza foi realizada nos domicílios. O profissional de saúde ficava na calçada, o paciente no domicílio e o procedimento era feito através da meia porta.

\section{Dispensação de medicamentos}

Os medicamentos especiais de alto custo estão sendo entregues nos domicílios dos pacientes. Os medicamentos regulares estão sendo entregues, em alguns casos e grupos específicos, nos domicílios e em outros casos foi desenhado um processo de agendamento para entrega de medicações nas unidades de APS com entrada e fluxo específico para a farmácia, com todos os controles e higienização.

\section{Atenção aos usuários com condições crônicas}

As equipes de APS atuam de maneira territorial e são responsáveis por sua população. Por isso, cada equipe tem os pacientes crônicos identificados, mapeados e estratificados por risco. Baseadas nessa estratificação, as equipes têm desenvolvido ações de acompanhamento e teleatendimento via aplicativos de mensagens. Nos casos de maior risco e dificuldade com o acesso à tecnologia são feitas visitas domiciliares agendadas.

\section{Atuação do ACS e do ACE}

Os ACS têm permanecido atuando nos territórios com visitas peridomiciliares. Em parte, fazem o acompanhamento dos pacientes por meio de aplicativos de mensagens. Além disso, os ACS participaram de um processo de Educação Permanente e receberam os Equipamento de Proteção Individual (EPI) 
necessários para continuar fazendo as visitas no território sem entrar nos domicílios, ficando na área peridomiciliar.

Inicialmente as ações do ACE no território foram suspensas, porém, começaram a surgir casos novos de dengue. Portanto, optou-se por retomar o trabalho, e os ACE voltaram no território com os EPI necessários para fazer a verificação dos espaços externos, terrenos baldios e orientação às famílias.

\section{Teleatendimento}

A Secretaria Municipal de Saúde tem trabalhado no último ano no processo de implementação de aplicações computacionais. Começaram a ser desenvolvidas aplicações computacionais adaptadas à realidade de Sobral. Foi desenhada uma plataforma integrada onde se faz a regulação entre a APS e a atenção especializada, bem como o acompanhamento do abastecimento de medicamentos e recentemente, uma interface de integração do Prontuário Eletrônico da APS com a atenção especializada e hospital municipal. Além disso, são desenvolvidas estratégias de matriciamento por meio de telesaúde ou telemedicina, potencializadas por meio de aplicativo de mensagens no contexto das equipes da APS.

\section{Monitoramento e telemonitoramento}

Os casos prováveis e os casos confirmados identificados tanto na rede pública, quanto na rede privada são notificados para a Secretaria Municipal de Saúde. Esses casos (suspeitos ou confirmados) e seus contatos são georreferenciados e essa informação é repassada para os gerentes nas UBS para que as equipes de atenção primária façam o monitoramento. As estratégias de monitoramento são singulares, dependem das condições do território e das populações.

Algumas UBS fazem esse processo principalmente online por teleatendimento. Em outras onde isso não é possível, porque as pessoas estão em condições de vulnerabilidade, de modo que não têm acesso a celular e internet. Nestes casos, o monitoramento é feito presencialmente por meio de visitas peridomiciliares, com o apoio do ACS, com os EPI e todos os cuidados necessários. Essa estratégia de visitas domiciliares nos territórios mais vulneráveis tem sido fundamental para o monitoramento.

Com essas estratégias de monitoramento capilarizadas na APS e diferenciadas segundo as condições do território têm se identificado mais rapidamente os casos que podem se agravar, podendo fazer o acompanhamento, solicitar exame de imagem e se for necessário transferilos para o hospital de referência para COVID-19. Esses processos de monitoramento são possíveis devido à alta cobertura da ESF no município. Característica que não é compartilhada pelos municípios vizinhos que apresentam percentuais de cobertura inferiores, o qual tem se refletido no agravamento dos casos nesses locais e o consequente aumento das internações dos municípios vizinhos.

Além disso, os CSF articulam-se com o Plantão Epidemiológico $24 \mathrm{~h}$ que se constitui como um canal de diálogo para os usuários do SUS. O plantão epidemiológico articula-se com a Central de Monitoramento da COVID-19 e o Comitê de Crise que compartilham situações específicas de usuários com os gerentes da APS, que planejam e desenvolvem juntos às equipes estratégias singulares de monitoramento e cuidado aos usuários inseridos nos territórios.

Atuação das equipes de saúde bucal e NASF Todos os atendimentos de saúde bucal não urgentes foram suspensos. Todas as urgências odontológicas foram centralizadas em uma Unidade de Pronto Atendimento (UPA). Tanto os profissionais de saúde bucal quanto os dos NASF estão apoiando os processos de monitoramento online ou presencial, dando suporte na organização das unidades de saúde e das tendas. Além disso, os profissionais dos NASF também estão realizando matriciamento com as equipes para os casos mais complexos. 


\section{Proteção e cuidado dos profissionais da saúde}

Do mesmo modo que em outros locais do Brasil, inicialmente houve dificuldades para aquisição de EPI. Porém, duas fábricas existentes em Sobral (uma de calçados e outra de cimento) mudaram toda organização produtiva e começaram a produzir EPI para o município. Esse processo tem contribuído muito para manter o suprimento adequado de EPI na APS. Para a organização de dispensação dos EPI foi estruturada uma Comissão de entrega de material que realiza o monitoramento e controle da dispensação e articula-se com o setor de articulação e aquisição de materiais para garantir a manutenção dos insumos necessários para o cuidado.

\section{Testes}

Inicialmente houve dificuldades para conseguir testes, mas recentemente foi adquirida uma quantidade considerável de testes rápidos. 0 governo do estado também comprou uma quantidade importante tanto de testes rápidos sorológicos, quanto para RT-PCR. Neste momento, por cada caso confirmado são feitos em torno de 20 testes rápidos para os contatos, sem importar se são sintomáticos ou assintomáticos. Isso com o objetivo de detectar mais precocemente os casos e instituir isolamento domiciliar.

Uma grande dificuldade que permanece é o tempo de retorno dos resultados dos testes de RT-PCR. No início, os resultados podiam tardar até 15-16 dias porque todas as mostras do estado estão sendo processadas no laboratório central em Fortaleza. Neste momento, o governo de estado está tentando descentralizar o laboratório e já conseguiu reduzir o tempo de resposta.

\section{COMPARTILHAMENTO DO CUIDADO EM}

\section{REDE}

O processo de compartilhamento do cuidado em rede foi potencializado com a situação de enfrentamento da pandemia, uma vez que a atenção especializa passou a fortalecer a APS com a incorporação de profissionais nas equipes de ESF e da ampliação das ações de monitoramento.

Como a APS é a ordenadora do cuidado e a porta de entrada preferencial para o desenvolvimento de ações e serviços ofertadas pelas Redes de Atenção à Saúde, ela ocupa o papel central no enfrentamento ao novo coronavírus (COVID-19) a partir da necessidade imediata de capilarização das informações e ações para o território.

Neste sentido, a linha de cuidado foi construída tendo como referência a compressão da APS como ordenadora da rede de atenção. Assim, a APS realiza o processo de identificação de sintomáticos respiratórios e a partir de então orienta e monitora o isolamento domiciliar, bem como a evolução dos sintomas e a articulação com postos de coleta de exames de Biologia molecular (RT-PCR em tempo real, detecção do vírus SARS-CoV2) e imunocromatográficos (teste rápido).

A APS acompanha os usuários do seu território sanitário e ao identificar a necessidade de compartilhamento do cuidado em rede com os demais serviços, dialoga com a atenção especializada para a realização de exames de imagem e com os hospitais e UPA para o processo de avaliação/internação.

Além disso, a participação comunitária e o controle social fortaleceram suas ações nos territórios com o trabalho dos Conselhos Locais de Saúde, bem como do processo de compartilhamento da tomada de decisão com o Conselho Municipal de Saúde. Neste sentido, a rede de atenção à saúde buscou incorporar em suas ações descentralizadas a colaboração das lideranças comunitárias a fim de sensibilizar a população sobre a importância do isolamento social para evitar a disseminação do vírus, bem como medidas de prevenção da COVID-19 e promoção da saúde. 
Salienta-se ainda, o desenvolvimento de processos de Educação Permanente em Saúde dos trabalhadores que integram a rede de serviços relacionados ao enfrentamento da COVID-19. Deste modo, foram utilizados recursos de educação à distância por meio da plataforma moodle.

Além disso, a Escola de Saúde Pública Visconde de Sabóia, junto com os docentes e residentes da Residência Multiprofissional em Saúde da Família e Residência Multiprofissional em Saúde Mental, desenvolveram plantões de escuta para profissionais da saúde e usuários, com o suporte dos psicólogos residentes, bem como, a realização de lives por meio das redes sociais Facebook e Instagram com temáticas relacionadas a prevenção da COVID-19 e promoção da saúde.

\section{CONSIDERAÇõES FINAIS}

A experiência de Sobral evidencia a importância do fortalecimento do Sistema Único de Saúde (SUS) e da APS, em especial do modelo assistencial ancorado na abordagem familiar e comunitária, territorializada e considerando a participação social no enfrentamento da COVID19, como coordenadora do cuidado em articulação com os demais pontos da rede de atenção à saúde e de outros dispositivos intersetoriais.

Neste sentido, a APS tem um papel estratégico no enfrentamento da COVID-19 de modo que em Sobral foram fortalecidas as ações de vigilância tendo como enfoque a perspectiva territorial para o desenvolvimento do cuidado em rede, além disso, as atividades próprias da APS foram ressignificadas por meio de adaptações nos serviços e ações ofertados aos usuários do SUS.

Assim, todo o investimento realizado na APS de Sobral nos últimos anos vem sendo fundamental no enfrentamento da COVID-19, uma vez que as estruturas físicas das UBS e o desenvolvimento/refinamento dos processos de trabalho colaboram para a construção de um cuidado colaborativo, ancorados na interprofissionalidade e intersetorialidade. Assim, a pandemia da COVID-19 deverá fortalecer os aprendizados acerca da APS como coordenadora da gestão do cuidado em rede. 


\section{NOTAS E REFERÊNCIAS}

BARBOSA, S. P.; SILVA, A. V. F. G. A Prática da Atenção Primária à Saúde no Combate da Covid-19. APS em Revista, v. 2, n. 1, p.17-19. 2020. Disponível em: https://apsemrevista.org/aps/article/view/62/43. Acesso em: 20 mai 2020.

BRASIL. Ministério da Saúde. Diretrizes para diagnóstico e tratamento da COVID-19. Brasília: Editora do Ministério da Saúde, 2020a.

BRASIL. Ministério da Saúde. Portaria no 188, de 3 de fevereiro de 2020. Declara Emergência em Saúde Pública de importância Nacional (ESPIN) em decorrência da Infecção Humana pelo novo Coronavírus (2019-nCoV). Brasília: Diário Oficial da União, 2020b.

BRASIL. Ministério da Saúde. Portaria no 454, de 20 de março de 2020. Declara, em todo o território nacional, o estado de transmissão comunitária do coronavírus (COVID-19). Brasília: Diário Oficial da União, 2020c.

BRASIL. Ministério da Saúde. Portaria no 1378/GM/MS, de 09 de julho de 2013. Regulamenta as responsabilidades e define diretrizes para execução e financiamento das ações de Vigilância em Saúde pela União, Estados, Distrito Federal e Municípios, relativos ao Sistema Nacional de Vigilância em Saúde e Sistema Nacional de Vigilância Sanitária. Brasília: Ministério da Saúde, 2013. Disponível em: https://bvsms.saude.gov.br/bvs/saudelegis/gm/2013/prt1378_09_07_2013.html. Acesso em: 02 mai. 2020.

CASCELLA, M.; RAJNIK, M.; CUOMO, A.; DULEBOHN, S. C.; DI NAPOLI, R. Features, evaluation and treatment coronavirus (COVID-19). In: StatPearls. Treasure Island (FL): StatPearls Publishing; 2020. Disponível em: https://www.ncbi.nlm.nih.gov/books/NBK554776/. Acesso em: 18 mai 2020.

CEARÁ. Secretaria de Saúde do Estado. Coordenadorias Regionais de Saúde. 2020a.

GORBALENYA, A.E., BAKER, S.C., BARIC, R.S. et al. The species Severe acute respiratory syndrome-related coronavirus: classifying 2019-nCoV and naming it SARS-CoV-2. Nat Microbiol, p. 536-544. 2020. Disponível em: Acesso em: 25 mai 2020.

MERHY, E, E. Saúde: a cartografia do trabalho vivo. 3a Ed. São Paulo: Editora Hucitec, 2002.

NEDEL, F. B. Enfrentando a COVID-19: APS forte agora mais que nunca! APS em revista, v. 2, n. 1, p.1116. 2020. Disponível em: https://apsemrevista.org/aps/article/view/68/42. Acesso em: 06 mai 2020.

OLIVEIRA, C. M.; CASANOVA, Â. O. Vigilância da saúde no espaço de práticas da atenção básica. Ciênc. saúde coletiva, Rio de Janeiro, v.14, n. 3, p. 929-936. $2009 . \quad$ Disponível em: http://www.scielo.br/scielo.php?script=sci_arttext\&pid=S1413-81232009000300029\&lng=en\&nrm=iso. Acesso em: 20 mai 2020.

ROLNIK, Suely. Cartografia sentimental: transformações contemporâneas do desejo. 2. ed. Sulina: Porto Alegre, 2014.

TUÑAS, I. T. C. et al. Doença pelo Coronavírus 2019 (COVID-19): Uma abordagem preventiva para Odontologia. Revista Brasileira de Odontologia, v.77, 2020. 
Carvalho, R. C.; Cunha, I. C. K. O.

WHO. WHO announces COVID-19 outbreak a pandemic. 2020 Disponível em: http://www.euro.who.int/en/health-topics/health-emergencies/coronavirus-covid-

19/news/news/2020/3/who-announces-covid-19-outbreak-a-pandemic. Acesso em: 19 mai 2020.

ZHANG, J-J et al. Clinical characteristics of 140 patients infected with SARS-CoV-2 in Wuhan, China. Allergy. 2020. 